Pacific Journal of Mathematic 


\title{
BOUNDARY POINTS OF JOINT NUMERICAL RANGES
}

\author{
Muneo Chō AND Makoto TakaguChI
}

\begin{abstract}
In this paper it is shown that the conical points of the joint numerical range belong to the joint spectrum. Moreover, we discuss the bare points and extreme points of the joint numerical ranges for the $n$-tuples of commuting normal operators and Toeplitz operators.
\end{abstract}

Introduction. The notion of the joint numerical range was first investigated by Halmos ([6], Prob. 166). Dash [4] tried to find how much of the knowledge about the numerical range in the single operator case carried over to the analogous situation in the case of an $n$-tuple of operators. Our purpose is to discuss the same subject as his. Dash [4] studied particularly about the convexity of the numerical range known as the Toeplitz-Hausdorff theorem. Here we shall, however, bring the boundary point of the numerical range into focus. In the case of a single operator, many authors have asserted the results referring to the relation between the numerical range and spectrum. Concerning these, Dash [4], Juneja [8], Abramov [1], Buoni and Wadhwa [3] have investigated the relation between the joint spectrum and joint numerical range. Abramov [1] has shown that the conical point of the closure of the joint numerical range of $A=\left(A_{1}, \cdots, A_{n}\right)$ belongs to the joint approximate point spectrum of $A$ in the case of the family $A$ consisting of self-adjoint operators. In $\S 1$, our result shall be given more clearly than Abramov's one even to the family of arbitrary operators, by means of Hildebrandt's technique [7]. In $\S 2$, we shall introduce a class of operator-families called joint normaloid. And, in $\S 3$, we shall discuss the bare points and extreme points of joint numerical ranges for the operator-families belonging to the joint normaloid.

Notation and definition. Throughout this paper, $H$ will be a complex Hilbert space with the scalar product (, ) and the norm $\|\cdot\|$, and all operators on $H$ will be assumed to be linear and bounded. Let $A=\left(A_{1}, \cdots, A_{n}\right)$ be an $n$-tuple of operators on $H$. The joint numerical range of $A$ is the subset $W(A)$ of the $n$-dimensional unitary space $C^{n}$ such that

$$
W(A)=\left\{\left(\left(A_{1} x, x\right), \cdots,\left(A_{n} x, x\right)\right): x \in H,\|x\|=1\right\} .
$$

In the case of $n=1$, it is the usual numerical range of an operator. 
We shall say that a point $z=\left(z_{1}, \cdots, z_{n}\right)$ of $C^{n}$ is in the joint approximate point spectrum $\sigma_{\pi}(A)$ of $A$ if there exists a sequence $\left\{x_{i}\right\}$ of unit vectors in $H$ such that

$$
\left\|\left(z_{k}-A_{k}\right) x_{i}\right\| \longrightarrow 0(i \longrightarrow \infty), k=1, \cdots, n .
$$

A point $z=\left(z_{1}, \cdots, z_{n}\right)$ will be called a joint eigenvalue of $A$ if there exists a nonzero eigenvector $x$ such that

$$
A_{k} x=z_{k} x, \quad k=1, \cdots, n .
$$

And a point $z=\left(z_{1}, \cdots, z_{n}\right)$ will be said to be in the joint residual spectrum $\sigma_{r}(A)$ of $A$ if there exists a nonzero vector $x$ such that

$$
A_{k}^{*} x=\overline{z_{k}} x, k=1, \cdots, n,
$$

where $\overline{z_{k}}$ denotes the complex conjugate of $z_{k}$. (Consult [5].)

Moreover, let $A=\left(A_{1}, \cdots, A_{n}\right)$ be an $n$-tuple of mutually commuting operators. And let $A^{\prime \prime}$ be the double commutant of $A$. Then we shall say that a point $z=\left(z_{1}, \cdots, z_{n}\right)$ of $C^{n}$ is in the joint spectrum $\sigma(A)$ of $A$ relative to $A^{\prime \prime}$ if

$$
\sum_{k=1}^{n} B_{k}\left(A_{k}-z_{k}\right) \neq I,
$$

for all $B_{1}, B_{2}, \cdots, B_{n}$ in $A^{\prime \prime}$, where $I$ denotes the identity operator. (Consult [5].)

\section{Conical points.}

Definition 1. Let a closed subset $K$ of $C^{n}$ be called a closed convex cone with vertex $(0, \cdots, 0)$ whenever $K$ satisfies the following properties:

$$
\begin{gathered}
K+K \subset K, \\
\alpha K \subset K \text { for all } \alpha \geqq 0, \\
K \cap(-K)=\{(0, \cdots, 0)\} .
\end{gathered}
$$

If, for $F \subset C^{n}$ and $z=\left(z_{1}, \cdots, z_{n}\right) \in F$, there exists a closed convex cone $K$ with vertex $(0, \cdots, 0)$ such that $F \subset K-z$, then we shall call the point $z$ a conical point of $F$.

TheOREM 1. Let $A=\left(A_{1}, \cdots, A_{n}\right)$ be an n-tuple of arbitrary operators. If $z=\left(z_{1}, \cdots, z_{n}\right)$ is a conical point of $\overline{W(A)}$ (throughout we shall use the bar symbol for closure), then $z$ belongs to the joint approximate point spectrum $\sigma_{\pi}(A)$ of $A$. If, moreover, $z$ is in $W(A)$, then $z$ is a joint eigenvalue of $A$. 
Proof. We may assume without loss of generality that the conical point $z$ of $\overline{W(A)}$ is $(0, \cdots, 0)$. Then we can choose $n$ linearly independent vectors $\boldsymbol{a}_{1}, \cdots, \boldsymbol{a}_{n}$ in $\boldsymbol{C}^{n}$ and $n$ constants $\theta_{1}, \cdots, \theta_{n}$ such that $0 \leqq \theta_{k}<\pi, k=1, \cdots, n$ and

$$
\overline{W(A}) \subset\left\{\alpha_{1} \boldsymbol{a}_{1}+\cdots+\alpha_{n} \boldsymbol{a}_{n}: 0 \leqq \arg \alpha_{k} \leqq \theta_{k}, k=1, \cdots, n\right\} .
$$

Let a set $\left\{\boldsymbol{e}_{1}, \cdots, \boldsymbol{e}_{n}\right\}$ of vectors in $\boldsymbol{C}^{n}$ be a basis in $\boldsymbol{C}^{n}$ such that the $j$ th coordinate of $\boldsymbol{e}_{k}$ is $\delta_{k j}, k=1, \cdots, n$, and $\boldsymbol{e}_{k}=\gamma_{1 k} \boldsymbol{a}_{1}+\cdots+$ $\gamma_{n k} \boldsymbol{a}_{n}, k=1, \cdots, n$. Putting

$$
B_{k}=\gamma_{k 1} A_{1}+\cdots+\gamma_{k n} A_{n}, \quad k=1, \cdots, n,
$$

it follows that

(*) $\overline{W\left(B_{1}, \cdots, B_{n}\right)} \subset\left\{\left(\beta_{1}, \cdots, \beta_{n}\right): 0 \leqq \arg \beta_{k} \leqq \theta_{k}, k=1, \cdots, n\right\}$.

We shall apply Hildebrandt's method [7, p. 232] to the argument follows. Let $k$ be any fixed element in the index set $\{1, \cdots, n\}$. We put here

$$
e^{i \vartheta_{k}}=\lambda_{k}=\mu_{k}+i \nu_{k},
$$

where $\mu_{k}, \nu_{k}$ are real numbers. Since we can, moreover, assume $\theta_{k}$ to be nonzero, $\nu_{k}$ is assumed to be nonzero. Therefore

$$
i=\frac{1}{\nu_{k}}\left(\lambda_{k}-\mu_{k}\right) \text {. }
$$

Furthermore, we decompose $B_{k}$ such that

$$
B_{k}=X_{k}+i Y_{k},
$$

where $X_{k}, Y_{k}$ are self-adjoint. Substituting the formula (**) for $i$,

$$
B_{k}=X_{k}-\frac{\mu_{k}}{\nu_{k}} Y_{k}+\lambda_{k}\left(\frac{1}{\nu_{k}} Y_{k}\right)
$$

Here, we put

$$
T_{k}=X_{k}-\frac{\mu_{k}}{\nu_{k}} Y_{k} \quad \text { and } \quad S_{k}=\frac{1}{\nu_{k}} Y_{k} .
$$

Then $T_{k}, S_{k}$ are self-adjoint and $B_{k}=T_{k}+\lambda_{k} S_{k}$. Since $\left(B_{k} x, x\right)=$ $\left(T_{k} x, x\right)+\lambda_{k}\left(S_{k} x, x\right)$ for every unit vector $x, T_{k}$ and $S_{k}$ are positive from $(*)$.

Now, since $z=(0, \cdots, 0) \in \overline{W(A}),(0, \cdots, 0) \in \overline{W(B})$ and then there exists a sequence $\left\{x_{i}\right\}$ of unit vectors such that $\left(B_{k} x_{i}, x_{i}\right) \rightarrow 0(k=1$, $\cdots, n, i \rightarrow \infty)$. And, since $\left(T_{k} x_{i}, x_{i}\right)$ and $\left(S_{k} x_{i}, x_{i}\right)$ also converge to zero for every $k$, we have 


$$
B_{k} x_{i} \longrightarrow 0(i \longrightarrow \infty), \quad k=1, \cdots, n \text {. }
$$

On the other hand, since the matrix $\boldsymbol{M}$ :

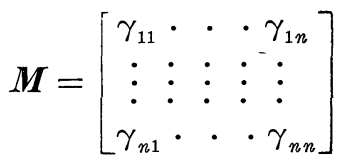

is regular,

$$
\left[\begin{array}{c}
A_{1} \\
\vdots \\
A_{n}
\end{array}\right]=\boldsymbol{M}^{-1}\left[\begin{array}{c}
B_{1} \\
\vdots \\
B_{n}
\end{array}\right] .
$$

Hence we have $A_{k} x_{i} \rightarrow 0(i \rightarrow \infty), k=1, \cdots, n$. Thus we get the proof of the first half of the theorem.

Next we assume that $z=(0, \cdots, 0) \in W(A)$. Then, there exists a unit vector $x$ such that $\left(A_{k} x, x\right)=0, k=1, \cdots, n$. So, if we take $x$ in place of the above sequence $\left\{x_{i}\right\}$, the proof of the latter half of the theorem follows in the same way as the first half.

\section{Joint normaloid operator-families.}

Definition 2. For any $n$-tuple $A=\left(A_{1}, \cdots, A_{n}\right)$ of operators, the following nonnegative numbers:

$$
\begin{aligned}
& \|A\|=\sup \left\{\left(\left\|A_{1} x\right\|^{2}+\cdots+\left\|A_{n} x\right\|^{2}\right)^{1 / 2}:\|x\|=1\right\}, \\
& w(A)=\sup \left\{\left(\left|\left(A_{1} x, x\right)\right|^{2}+\cdots+\left|\left(A_{n} x, x\right)\right|^{2}\right)^{1 / 2}:\|x\|=1\right\}, \\
& r(A)=\sup \left\{\left(\left|z_{1}\right|^{2}+\cdots+\left|z_{n}\right|^{2}\right)^{1 / 2}: z \in \sigma(A)\right\}, \\
& r_{\pi}(A)=\sup \left\{\left(\left|z_{1}\right|^{2}+\cdots+\left|z_{n}\right|^{2}\right)^{1 / 2}: z \in \sigma_{\pi}(A)\right\}
\end{aligned}
$$

are called the joint operator norm, joint numerical radius, joint spectral radius and joint approximate point spectral radius respectively, of $A$.

Definition 3. An $n$-tuple $A=\left(A_{1}, \cdots, A_{n}\right)$ of operators is said to belong to the joint normaloid or to be joint normaloid if $w(A)=$ $\|A\|$.

In order to show the following propositions we need the following results shown by Dash [5].

First, suppose that $A=\left(A_{1}, \cdots, A_{n}\right)$ is a commuting $n$-tuple of normal operators. Then there exists a measure space $(X ; \mu)$ and a set of bounded measurable functions $\phi_{1}, \cdots, \phi_{n}$ in $L^{\infty}(X ; \mu)$ such that each $A_{k}$ is unitary equivalent to the multiplication by $\phi_{k}$ on $L^{2}(X ; \mu)$, 
$k=1, \cdots, n$. That is,

$$
A_{k} f=\phi_{k} f \quad \text { for all } f \in L^{2}(X ; \mu), k=1, \cdots, n .
$$

And the joint spectrum of $A$ is the joint essential range of $\phi=$ $\left(\phi_{1}, \cdots, \phi_{n}\right)$, that is, the set of all the points $z=\left(z_{1}, \cdots, z_{n}\right)$ in $C^{n}$ such that for every $\varepsilon>0$

$$
\mu\left(\left\{t \in X: \sum_{k=1}^{n}\left|\phi_{k}(t)-z_{k}\right|<\varepsilon\right\}\right)>0 .
$$

And the joint spectrum of $A$ is equal to the joint approximate point spectrum of $A$, i.e., $\sigma(A)=\sigma_{\pi}(A)$.

Secondly, suppose that $\phi=\left(\phi_{1}, \cdots, \phi_{n}\right)$ is an $n$-tuple of bounded measurable function on the unit circle and $L_{\phi}=\left(L_{\phi_{1}}, \cdots, L_{\phi_{n}}\right)$ and $T_{\phi}=\left(T_{\phi_{1}}, \cdots, T_{\phi_{n}}\right)$ are the $n$-tuples of the Laurent operators on $L^{2}$ and Toeplitz operators on $H^{2}$ respectively induced by $\phi$. That is, for each $k=1, \cdots, n$,

$$
L_{\phi_{k}} f=\phi_{k} f \text { for all } f \in L^{2} \text {, and } T_{\phi_{k}} f=P L_{\phi_{k}} f \text { for all } f \in H^{2},
$$

where $P$ denotes the projection from $L^{2}$ onto $H^{2}$. Then the joint spectrum of $L_{\phi}$ is a subset of the joint approximate point spectrum of $T_{\phi}$, i.e., $\sigma\left(L_{\phi}\right) \subset \sigma_{\pi}\left(T_{\phi}\right)$. If, furthermore, all $T_{\phi_{k}}, k=1, \cdots, n$, are analytic, i.e., all $\phi_{k}$ belong to $H^{\infty}$, then the joint spectrum of $T_{\phi}$ is the closure of the joint residual spectrum of it. (Consult Dash [5].)

Proposition 1. If $A=\left(A_{1}, \cdots, A_{n}\right)$ is a commuting $n$-tuple of normal operators, then $\|A\|=w(A)=r(A)$, and so $A$ is joint normaloid.

Proof. Since $\sigma(A)=\sigma_{\pi}(A)$, it follows that $w(A) \geqq r(A)$. On the other hand, it follows that

$$
\|A\|^{2}=\sup \left\{\int_{k=1}^{n}\left|\phi_{k}(t)\right|^{2}|f(t)|^{2}(d \mu)(t):\|f\|=1\right\}
$$

and

$$
r(A)^{2}=\sup \left\{\sum_{k=1}^{n}\left|z_{k}\right|^{2}: \mu\left(\left\{t \in X: \sum_{k=1}^{n}\left|\phi_{k}(t)-z_{k}\right|<\varepsilon\right\}\right)>0 \text { for any } \varepsilon>0\right\},
$$

from the definition and the above Dash's results. Since

$$
\mu\left(\left\{t \in X: \sum_{k=1}^{n}\left|\phi_{k}(t)\right|^{2}>r(A)^{2}\right\}\right)=0,
$$

it follows that 


$$
\int \sum_{k=1}^{n}\left|\phi_{k}(t)\right|^{2}|f(t)|^{2}(d \mu)(t) \leqq r(A)^{2} \cdot \int|f|^{2} d \mu \leqq r(A)^{2}\|f\|^{2} .
$$

Hence $\|A\| \leqq r(A)$. So, the proof is complete.

Proposition 2. If $T_{\phi}=\left(T_{\phi_{1}}, \cdots, T_{\phi_{n}}\right)$ is an n-tuple of Toeplitz operators, then $\left\|T_{\phi}\right\|=w\left(T_{\phi}\right)=r_{\pi}\left(T_{\phi}\right)$, and so $T_{\phi}$ is joint normaloid. Moreover, if $T_{\phi}$ is an n-tuple of analytic Toeplitz operators, then $\left\|T_{\phi}\right\|=w\left(T_{\phi}\right)=r\left(T_{\phi}\right)$.

Proof. It holds that $\left\|T_{\phi}\right\| \leqq\left\|L_{\phi}\right\| \leqq r\left(L_{\phi}\right) \leqq r_{\pi}\left(T_{\phi}\right) \leqq w\left(T_{\phi}\right) \leqq$ $\left\|T_{\phi}\right\|$. So, the first half is proved. Moreover, if $T_{\phi_{1}}, \cdots, T_{\phi_{n}}$ are all analytic, then $\sigma\left(T_{\phi}\right)=\overline{\sigma_{r}\left(T_{\phi}\right)} \subset \overline{W\left(T_{\phi}\right)}$. Hence

$$
r_{\pi}\left(T_{\phi}\right) \leqq r\left(T_{\phi}\right) \leqq w\left(T_{\phi}\right),
$$

and so the latter half is proved, too.

3. The bare points and extreme points of the joint normaloid operator-family.

DeFinition 4. Let $K$ be a bounded and connected set in $C^{n}$. The point $\alpha$ of $K$ will be called an extreme point of $K$ if no line segment joining any two points of $K$ other than $\alpha$ contains $\alpha$. And the point $\beta$ of $K$ will be called a bare point of $K$ if there exists a spherical surface through $\beta$ such that no points of $K$ lie outside this spherical surface.

The set of the bare points of $K$ is included in the set of extreme points of $K$ and dense in it (cf. Berberian [2], p. 181).

TheOREM 2. Let $A=\left(A_{1}, \cdots, A_{n}\right)$ be an n-tuple of operators such that $A-z=\left(A_{1}-z_{1}, \cdots, A_{n}-z_{n}\right)$ is joint normaloid for every point $z=\left(z_{1}, \cdots, z_{n}\right)$ in $C^{n}$. If $\alpha=\left(\alpha_{1}, \cdots, \alpha_{n}\right)$ is an extreme point of $\overline{W(A)}$, then $\alpha$ belongs to the joint approximate point spectrum $\sigma_{\pi}(A)$ of $A$. If, moreover, $\alpha$ is a bare point of $W(A)$, then $\alpha$ is a joint eigenvalue of $A$.

Proof. Observing the joint approximate point spectrum is closed, it is sufficient for the proof to show that $\alpha \in \sigma_{\pi}(A)$ if $\alpha$ is a bare point of $\overline{W(A)}$. So, now, let $\alpha$ be a bare point of $\overline{W(A)}$. Then there exists a spherical surface $S$ with the central point $z=\left(z_{1}, \cdots, z_{n}\right)$ such that no points of $\overline{W(A)}$ lie outside $S$ and $\alpha$ is on $S$. Thus,

$$
\left(\sum_{k=1}^{n}\left|\alpha_{k}-z_{k}\right|^{2}\right)^{1 / 2}=w(A-z)=\|A-z\|
$$


and there exists a sequence $\left\{x_{i}\right\}$ of unit vectors such that

$$
\left(\left(A_{k}-z_{k}\right) x_{i}, x_{i}\right) \longrightarrow \alpha_{k}-z_{k}(i \longrightarrow \infty), k=1, \cdots, n \text {. }
$$

Consequently,

$$
\begin{aligned}
\sum_{k=1}^{n} \| & \left(A_{k}-\alpha_{k}\right) x_{i}\left\|^{2}=\sum_{k=1}^{n}\right\|\left(A_{k}-z_{k}\right) x_{i}-\left(\alpha_{k}-z_{k}\right) x_{i} \|^{2} \\
= & \sum_{k=1}^{n}\left\|\left(A_{k}-z_{k}\right) x_{i}\right\|^{2}-2 \cdot \operatorname{Re} \sum_{k=1}^{n}\left(\overline{\alpha_{k}}-\overline{z_{k}}\right)\left(\left(A_{k}-z_{k}\right) x_{i}, x_{i}\right) \\
& +\sum_{k=1}^{n}\left|\alpha_{k}-z_{k}\right|^{2} \longrightarrow 0 \quad(i \longrightarrow \infty) .
\end{aligned}
$$

Hence $\alpha \in \sigma_{\pi}(A)$. The latter half is also proved in the same way as the first half.

REMARK. In the case of a single operator, if $A-z$ is normaloid for every complex number $z$, Hildebrandt [7] said $A$ to belong to operator-class $C_{3}$.

Corollary 1. For any n-tuple $A=\left(A_{1}, \cdots, A_{n}\right)$ of operators, $w(A)=\|A\|$ if and only if $r_{\pi}(A)=\|A\|$.

Corollary 2. Let $A=\left(A_{1}, \cdots, A_{n}\right)$ be an n-tuple of commuting normal operators. If $\alpha$ is an extreme point of $\overline{W(A})$, then $\alpha \in \sigma_{\pi}(A)$. If $\alpha$ is a bare point of $W(A)$, then $\alpha$ is a joint eigenvalue of $A$.

Corollary 3. Let $A=\left(A_{1}, \cdots, A_{n}\right)$ be an n-tuple of Toeplitz operators. If $\alpha$ is an extreme point of $\overline{W(A})$, then $\alpha \in \sigma_{\pi}(A)$.

Dash [4] has shown that $W(A)$ is convex, if $A=\left(A_{1}, \cdots, A_{n}\right)$ is a commuting $n$-tuple of normal operators or an $n$-tuple of Toeplitz operators (see Thm. 2.5 and Thm. 2.6 in [4]). Now, we recall that $\left.\sigma(A)=\sigma_{\pi}(A) \subset \overline{W(A}\right)$ if $A$ is a commuting $n$-tuple of normal operators. And if $T_{\phi}$ is an $n$-tuple of analytic Toeplitz operators, then $\sigma\left(T_{\phi}\right)=$ $\overline{\sigma_{r}\left(T_{\phi}\right)} \subset \overline{W\left(T_{\phi}\right)}$. Consequently, we get the followings.

Corollary 4 ([4], Thm. 2.8). Let $A=\left(A_{1}, \cdots, A_{n}\right)$ be a commuting n-tuple of normal operators. Then we have

$$
\sum(\sigma(A))=\overline{W(A)},
$$

where $\sum(\sigma(A))$ denotes the convex hull of $\sigma(A)$.

CoRollary 5 ([4], Thm. 2.10). Let $T_{\phi}=\left(T_{\phi_{1}}, \cdots, T_{\phi_{n}}\right)$ be an 
n-tuple of Toeplitz operators. Then we have

$$
\sum\left(\sigma_{\pi}\left(T_{\dot{\phi}}\right)\right)=\overline{W\left(T_{\dot{\phi}}\right)} .
$$

CoRollary 6 ([4], Cor. 2.11). Let $T_{\phi}=\left(T_{\phi_{1}}, \cdots, T_{\phi_{n}}\right)$ be an $n$ tuple of analytic Toeplitz operators. Then we have

$$
\left.\sum\left(\sigma\left(T_{\phi}\right)\right)=\overline{W\left(T_{\phi}\right.}\right) .
$$

In the case of single operators, Klein [9] has shown that the numerical range of a Toeplitz operator has no extreme points if it is nonconstant. Next, we shall generalize his result for the case of operator-families.

Proposition 3. Let $T_{\phi}=\left(T_{\phi_{1}}, \cdots, T_{\phi_{n}}\right)$ be an n-tuple of Toeplitz operators. Unless the joint numerical range of $T_{\phi}$ consists of only one point, it has no extreme points and so it is an open set.

Proof. Now, suppose that there exists an extreme point in $W\left(T_{\phi}\right)$ and that $z=\left(z_{1}, \cdots, z_{n}\right)$ is its point. Then there exists an $n \times n$ unitary matrix $\boldsymbol{U}$ :

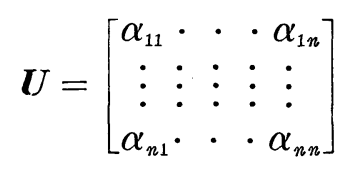

such that the point $\alpha_{11} z_{1}+\alpha_{12} z_{2}+\cdots+\alpha_{1 n} z_{n}$ in $C$ is the extreme point of the numerical range of the operator $T \equiv \alpha_{11} T_{\phi_{1}}+\alpha_{12} T_{\phi_{2}}+$ $\cdots+\alpha_{1 n} T_{\phi_{n}}$ and $T$ is nonconstant. So, since the operator $T$ is also Toeplitz, it is impossible from Klein's results. Therefore, the joint numerical range of $T_{\phi}$ has no extreme points.

We would like to express our thanks to the referee for his useful comments.

\section{REFERENCES}

1. Y. S. Abramov, On the geometric properties of the joint spectrum of a family of self-adjoint operators, Studia Math., 61 (1977), 55-62.

2. S. K. Berberian, Lectures in Functiona! Analysis and Operator Theory, SpringerVerlag, New York, 1974.

3. J. J. Buoni and B. L. Wadhwa, On joint numerical ranges, Pacific J. Math., 77 (1978), 303-306.

4. A. T. Dash, Joint numerical range, Glasnik Mat., 7 (1972), 75-81.

$5 . \quad$ J Joint spectra, Studia Math., 45 (1973), 225-237.

6. P. R. Halmos, A Hilbert Space Problem Book, Van Nostrand, Princeton, 1967.

7. S. Hildebrandt, Über den numerischen Wertebereich eines Operators, Math. Ann., 163 (1966), 230-247. 
8. P. Juneja, On extreme points of the joint numerical range of commuting normal operators, Pacific J. Math., 67 (1976), 473-476.

9. E. M. Klein, The numerical range of a Toeplitz operator, Proc. Amer. Math. Soc., 35 (1972), 101-103.

Received August 6, 1979 and in revised form March 12, 1980.

Department of Mathematics

FACULTY OF SCIENCE

HIROSAKI UNIVFRSITY

HIROSAKI 036

JAPAN 



\section{PACIFIC JOURNAL OF MATHEMATICS}

\section{EDITORS}

DONALD BABBITT (Managing Editor)

University of Galifornia

Los Angeles, California 90024

Hugo RossI

University of Utah

Salt Lake City, UT 84112

C. C. MOORE AND ANDREW OGG

University of California

Berkeley, CA 94720
J. DugundJI

Department of Mathematics University of Southern California Los Angeles, California 90007

R. FinN and J. Milgram Stanford University Stanford, California 94305

\section{ASSOCIATE EDITORS}

R. ARENS

E. F. BeCKenbaCh

B. H. NEUManN

F. WOLF

K. YosHIDA

\section{SUPPORTING INSTITUTIONS}

UNIVERSITY OF ARIZONA

UNIVERSITY OF BRITISH COLUMBIA

CALIFORNIA INSTITUTE OF TECHNOLOGY

UNIVERSITY OF CALIFORNIA

MONTANA STATE UNIVERSITY

UNIVERSITY OF NEVADA, RENO

NEW MEXICO STATE UNIVERSITY

OREGON STATE UNIVERSITY
UNIVERSITY OF OREGON

UNIVERSITY OF SOUTHERN CALIFONIA

STANFORD UNIVERSITY

UNIVERSITY OF HAWAII

UNIVERSITY OF TOKYO

UNIVERSITY OF UTAH

WASHINGTON STATE UNIVERSITY

UNIVERSITY OF WASHINGTON 


\section{Pacific Journal of Mathematics}

\section{Vol. 95, No. $1 \quad$ September, 1981}

John Allen Beachy and William David Blair, On rings with bounded

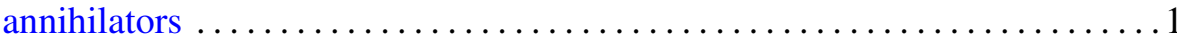

Douglas S. Bridges, A constructive look at positive linear functionals on

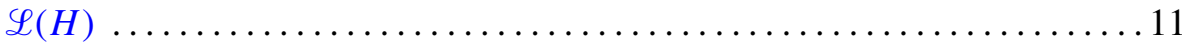

Muneo Chō and Makoto Takaguchi, Boundary points of joint numerical ranges

W. J. Cramer and William O. Ray, Solvability of nonlinear operator equations

Lester Eli Dubins and Gideon Schwarz, Equidiscontinuity of

Borsuk-Ulam functions

Maria Fragoulopoulou, Spaces of representations and enveloping 1.m.c.

*-algebras

Robert F. Geitz and J. Jerry Uhl, Jr., Vector-valued functions as families of scalar-valued functions

Ross Geoghegan, The homomorphism on fundamental group induced by a homotopy idempotent having essential fixed points

Ross Geoghegan, Splitting homotopy idempotents which have essential fixed points

Paul Jacob Koosis, Entire functions of exponential type as multipliers for

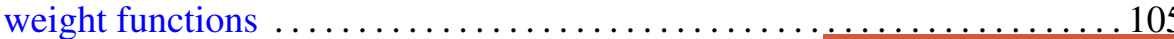

David London, Monotonicity of permanents of certain doubly stochastic matrices

Howard J. Marcum, Two results on cofibers

Giancarlo Mauceri, Zonal multipliers on the Heisenberg group

Edward Wilfred Odell, Jr. and Y. Sternfeld, A fixed point theorem in $c_{0} \quad \ldots 161$

Bernt Karsten Oksendal, Brownian motion and sets of harmonic measure zero

Andrew Douglas Pollington, The Hausdorff dimension of a set of normal numbers

Joe Repka, Base change lifting and Galois invariance ...

Gerald Suchan, Concerning the minimum of permanents on doubly stochastic circulants

Jun-ichi Tanaka, On isometries of Hardy spaces on compact abelian groups

Aaron R. Todd, Quasiregular, pseudocomplete, and Baire spaces 